\title{
Three versus five lumbar PVB injections for herniorrhaphy
}

\author{
Zoher Naja ${ }^{1}$ - Saleh Kanawati ${ }^{1} \cdot$ Fouad Ziade $^{2}$ \\ Received: 18 March 2019 / Accepted: 23 March 2019 / Published online: 5 April 2019 \\ (c) Japanese Society of Anesthesiologists 2019
}

Keywords Nerve block $\cdot$ Postoperative pain $\cdot$ Geriatric anesthesia

To the Editor:

We would like to thank Doctors Hu, Zhou and Zou for their comments regarding our paper that compared three versus five paravertebral injections for inguinal hernia repair in the elderly.

Regarding the analysis of the number of failed block between the two groups, when we applied Fisher's exact test (one-sided) hypothesizing the superiority of five injections over three injections, the resulting $p$ value was 0.03 which indicated a statistical significant difference.

As for bupivacaine, patients who received three paravertebral injections were given $15 \mathrm{ml}$ bupivacaine with a total dose of $75 \mathrm{mg}$. Similarly, patients who received five injections were given a total of $15 \mathrm{ml}$ bupivacaine with a total dose of $75 \mathrm{mg}$ (Fig. 1 in the paper) [1]. This was done to ensure double blindness and that the lumbar plexus in both groups received the same dose of bupivacaine although with different concentrations depending on the number of injections. Even though group 5 received higher bupivacaine concentration than group 5; yet, group 5 had more favorable outcome since it required less intraoperative supplemental analgesia and provided lower postoperative pain scores. This indicates that spread is not guaranteed in the lumbar plexus.

\section{Reference}

1. Naja Z, Kanawati S, El Khatib Z, Ziade F, Nasreddine R, Naja AS. Three versus five lumbar paravertebral injections for inguinal hernia repair in the elderly: a randomized double-blind clinical trial. J Anesth. 2019;33:50-7.

Publisher's Note Springer Nature remains neutral with regard to jurisdictional claims in published maps and institutional affiliations.

This reply refers to the comment available online at https://doi. org/10.1007/s00540-019-02636-2.

Zoher Naja

zouhnaja@yahoo.com

1 Anesthesia and Pain Management Department, Makassed

General Hospital, Beirut, Lebanon

2 Faculty of Public Health, Lebanese University, Beirut,

Lebanon 